\title{
Local White Matter Geometry Indices from Diffusion Tensor Gradients
}

\author{
Peter Savadjiev ${ }^{1,2}$, Gordon Kindlmann ${ }^{3}$, Sylvain Bouix ${ }^{1}$, Martha E. Shenton ${ }^{1}$, \\ and Carl-Fredrik Westin ${ }^{2}$ \\ ${ }^{1}$ Department of Psychiatry and ${ }^{2}$ Department of Radiology \\ Brigham and Women's Hospital, Harvard Medical School, Boston, MA, USA \\ ${ }^{3}$ Department of Computer Science, University of Chicago, Chicago, IL, USA
}

\begin{abstract}
We introduce a framework for computing geometrical properties of white matter fibres directly from diffusion tensor fields. The key idea is to isolate the portion of the gradient of the tensor field corresponding to local variation in tensor orientation, and to project it onto a coordinate frame of tensor eigenvectors. The resulting eigenframe-centered representation makes it possible to define scalar geometrical measures that describe the underlying white matter fibres, directly from the diffusion tensor field and its gradient, without requiring prior tractography. We define two new scalar measures of (1) fibre dispersion and (2) fibre curving, and we demonstrate them on synthetic and in-vivo datasets. Finally, we illustrate their applicability in a group study on schizophrenia.
\end{abstract}

\section{Introduction}

Despite the advent of high angular resolution diffusion imaging techniques, diffusion tensor (DT) data continues to be commonly acquired and utilized in a variety of studies in neuroscience and medicine, in particular in clinical settings. Empirically established connections between biological tissue properties and diffusion tensor measures exist. Such tensor measures include e.g. fractional anisotropy (FA), orientation etc., and have been the topic of several studies (e.g. [12]). However, few methods consider the differential structure of tensor properties, as introduced in e.g. 3. In this article we focus on tensor orientation, and perform a differential analysis of diffusion tensor fields which leads to novel methods for the recovery of a variety of white matter geometrical measures, or indices. By isolating the portion of the gradient of the tensor field corresponding to local variation in tensor orientation, and by projecting it onto a coordinate frame of tensor eigenvectors, we achieve an eigenframe-centered representation of local tensor field configurations. Given that the principal eigenvector generally represents the dominant orientation of the underlying fibre population 4, this allows us to define measures of fibre curving and fibre dispersion. The advantage of this approach is the recovery of fibre geometry measures directly from the tensor field, without requiring prior tractography as in e.g. [5].

As scalar measures, these indices can be used to study local fibre organisation in the context of population studies. As a proof-of-concept, we carry out a 
group study which finds certain differences in local fibre geometry between patients with schizophrenia and normal controls. Other potential applications, to be pursued in future work, include their use in tractography (e.g. as geometrical priors), as well as in a context of registration as geometrical landmarks.

\section{Mathematical Model}

\subsection{Rotation Tangents: Background}

Our method is based on the mathematical framework developed in 6 . Let $\boldsymbol{F}$ : $\mathbb{R}^{3} \mapsto \mathrm{Sym}_{3}$ be a diffusion tensor field, such that $\boldsymbol{F}(\boldsymbol{x})=\boldsymbol{D}$. Here $\mathrm{Sym}_{3}$ denotes the set of symmetric tensors $\left(\boldsymbol{D}=\boldsymbol{D}^{\top}\right)$ in $\mathbb{R}^{3} \otimes \mathbb{R}^{3}$. Consider the tensor rotation function $\psi$ which rotates $\boldsymbol{D}$ with rotation matrix $\boldsymbol{R} \in \mathrm{SO}_{3}$, the group of rotations on $\mathbb{R}^{3}$ :

$$
\psi(\boldsymbol{R}, \boldsymbol{D})=\boldsymbol{R} \boldsymbol{D} \boldsymbol{R}^{\top}
$$

In [6], the rotation tangent $\boldsymbol{\Phi}_{i}(\boldsymbol{D})$ associated with eigenvector $\boldsymbol{e}_{i}$ of diffusion tensor $\boldsymbol{D}$ is defined as the change of tensor value due to infinitesimal rotations around $\boldsymbol{e}_{i}$ :

$$
\boldsymbol{\Phi}_{i}(\boldsymbol{D})=\left.\frac{\partial \psi\left(\boldsymbol{R}_{\boldsymbol{e}_{i}}(\phi), \boldsymbol{D}\right)}{\partial \phi}\right|_{\phi=0},
$$

where $\boldsymbol{R}_{\boldsymbol{e}_{i}}(\phi)$ denotes rotation by angle $\phi$ around $\boldsymbol{e}_{i}$. The rotation tangent $\boldsymbol{\Phi}_{i}(\boldsymbol{D})$ is a second order gradient tensor onto which the tensor field gradient can be projected, in order to obtain three spatial gradients of orientation [6]:

$$
\nabla \widehat{\phi}_{i}(\boldsymbol{x})=\widehat{\boldsymbol{\Phi}}_{i}(\boldsymbol{F}(\boldsymbol{x})): \nabla \boldsymbol{F}(\boldsymbol{x})
$$

Here ":" is the tensor contraction operator (analogous to the vector dot product), $\widehat{\boldsymbol{\Phi}}_{i}$ are unit-norm rotation tangents, and $\nabla \widehat{\phi}_{i}$ are vectors that indicate in $\mathbb{R}^{3}$ the direction in which the tensor orientation around eigenvector $\boldsymbol{e}_{i}$ varies the fastest. In our work, we compute tensor field gradients as described in [6], by convolving the tensor field with partial derivative kernels of a uniform cubic B-spline.

\subsection{Scalar Geometric Measures}

The work presented in [7] argues for the representation of white matter fibre geometry (and that of sets of 3D curves in general) in terms of local coordinate frames. The idea is to capture the differential geometry of $3 \mathrm{D}$ curves by measuring changes in the tangent vector orientation in three mutually orthogonal directions provided by the tangent, normal and bi-normal vectors of a local coordinate frame. The projection of the change of tangent vector orientation in these three directions results in three curvature functions which characterize locally the differential geometry of 3D curve sets.

Motivated by this approach, in this paper we consider the projection of the three $\nabla \widehat{\phi}_{i}(3)$ into the local coordinate frame provided by the tensor eigenvectors. One can form a total of nine such projections $\nabla \widehat{\phi}_{i} \cdot \boldsymbol{e}_{j}, i, j \in\{1,2,3\}$. We 


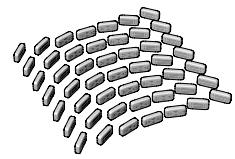

(a) $\nabla \widehat{\phi}_{2} \cdot e_{1}$

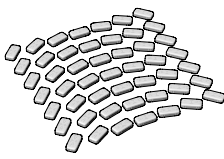

(b) $\nabla \widehat{\phi}_{3} \cdot e_{1}$

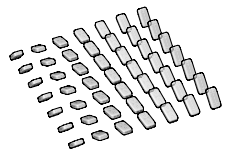

(c) $\nabla \widehat{\phi}_{2} \cdot \boldsymbol{e}_{2}$

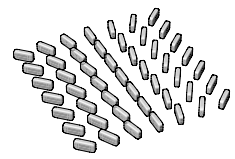

(d) $\nabla \widehat{\phi}_{2} \cdot e_{3}$

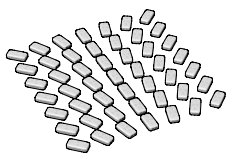

(e) $\nabla \widehat{\phi}_{3} \cdot e_{2}$

Fig. 1. Examples of local tensor field configurations that are characterized by a particular projection of the form $\nabla \widehat{\phi}_{i} \cdot \boldsymbol{e}_{j}$

choose the eigenframe as a projection basis due to the biological significance of tensor eigenvectors. It is commonly accepted that the principal eigenvector is aligned with the underlying white matter fibre tract in voxels where the diffusion anisotropy is strong enough (e.g. 4). We can thus relate patterns of tensor organisation to the geometry of white matter fibres.

Given the assumption that $\boldsymbol{e}_{1}$ represents the local fibre tangent direction, one can establish correspondences between observed tensor configurations and local fibre configurations. For example, $\nabla \widehat{\phi}_{2} \cdot \boldsymbol{e}_{1}$ and $\nabla \widehat{\phi}_{3} \cdot \boldsymbol{e}_{1}$ measure tangential change in tensor orientation (i.e. change in the tensor field seen by an observer displacing locally in the direction parallel to $\boldsymbol{e}_{1}$ ), which is analogous to the classical Frenet curvature, or the tangential curvature [7, of the underlying fibre. On the other hand, the projection of $\nabla \widehat{\phi}_{2}$ and $\nabla \widehat{\phi}_{3}$ in the plane spanned by $\boldsymbol{e}_{2}$ and $\boldsymbol{e}_{3}$ measures fibre orientation changes in directions orthogonal to $\boldsymbol{e}_{1}$. This is analogous to the normal and bi-normal curvatures of [7. Finally, tensor rotations around $e_{1}$, captured by $\nabla \widehat{\phi}_{1}$, would correspond to fibre twist.

Figure1illustrates a variety of local tensor configurations characterized by orientation change, consisting in tensor rotation around an eigenvector $\mathbf{e}_{i}$ observed in the direction of another eigenvector $\mathbf{e}_{j}$. For each example configuration, the projection $\nabla \widehat{\phi}_{i} \cdot \mathbf{e}_{j}$ will result in a high value (the specific values for $i$ and $j$ are indicated for each case).

Figure 2 illustrates these projections on a 2D synthetic diffusion tensor field with gradients in tensor orientation. Note that the projections are dependent on tensor shape. For instance, rotations around $\boldsymbol{e}_{1}$ do not result in significant change with nearly cylindrical tensors (i.e. $\lambda_{2}=\lambda_{3}$ ), hence $\nabla \widehat{\phi}_{1} \cdot \boldsymbol{e}_{1}$ gives smaller values in the bottom left part of Fig. 2(b) as the tensors become more cylindrical. Similarly, as the tensors approach a disk shape (i.e. $\lambda_{2}=\lambda_{3}$ ), rotation around $\boldsymbol{e}_{3}$ loses any effect, and $\nabla \widehat{\phi}_{3}$ and its eigenframe projections go to zero, as can be seen in the upper right part of Fig. 2(d).

We formalize the above observations by defining a local scalar index of fibre curving, $\mathcal{C}$, and a local scalar index of fibre dispersion, $\mathcal{D}$ :

$$
\text { Fibre curving: } \mathcal{C}(\boldsymbol{D}, \boldsymbol{x})=\sqrt{\left(\nabla \widehat{\phi}_{2} \nabla \widehat{\phi}_{2}^{\top}+\nabla \widehat{\phi}_{3} \nabla \widehat{\phi}_{3}^{\top}\right):\left(\boldsymbol{e}_{1} \boldsymbol{e}_{1}^{\top}\right)}
$$

Fibre dispersion: $\mathcal{D}(\boldsymbol{D}, \boldsymbol{x})=\sqrt{\left(\nabla \widehat{\phi}_{2} \nabla \widehat{\phi}_{2}^{\top}+\nabla \widehat{\phi}_{3} \nabla \widehat{\phi}_{3}^{\top}\right):\left(\boldsymbol{e}_{2} \boldsymbol{e}_{2}^{\top}+\boldsymbol{e}_{3} \boldsymbol{e}_{3}^{\top}\right)}$ 


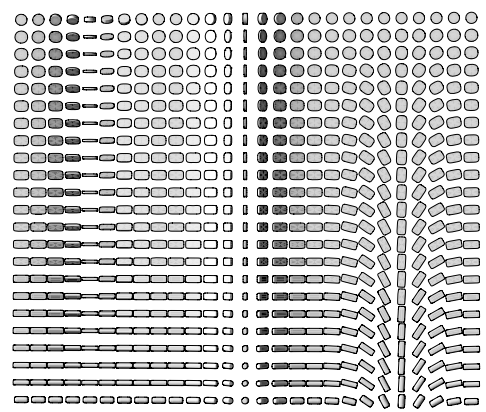

(a)

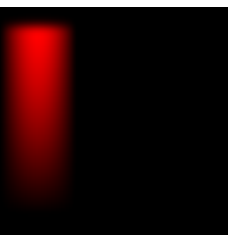

(b) $i=1$

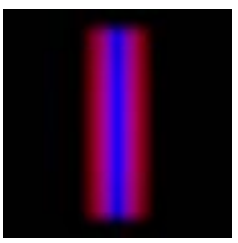

(c) $i=2$

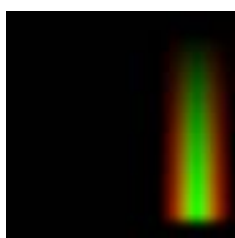

(d) $i=3$

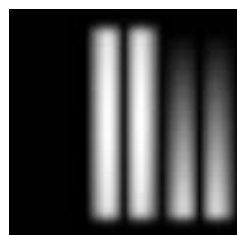

(e) $\mathcal{C}$

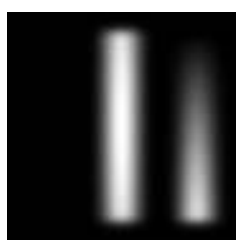

(f) $\mathcal{D}$

Fig. 2. (a) A 2D synthetic diffusion tensor field. (b,c,d) Results of projecting $\nabla \widehat{\phi}_{i}$ onto the local tensor eigenframe. Red: $\nabla \widehat{\phi}_{i} \cdot e_{1}$. Green: $\nabla \widehat{\phi}_{i} \cdot e_{2}$. Blue: $\nabla \widehat{\phi}_{i} \cdot e_{3}$. (e) Curving index. (f) Dispersion index. In (b)-(f), pixel intensity is proportional to index value.

The indices in (44) and (5) combine the rotation tangents $\nabla \widehat{\phi}_{2}$ and $\nabla \widehat{\phi}_{3}$ into a single second order tensor $\left(\nabla \widehat{\phi}_{2} \nabla \widehat{\phi}_{2}^{\top}+\nabla \widehat{\phi}_{3} \nabla \widehat{\phi}_{3}^{\top}\right)$, which is then projected onto another second order tensor that represents the fibre tangent direction in the case of the curving measure (4), or the plane orthogonal to that direction in the case of (5). The use of outer products and tensor contractions sidesteps the sign ambiguity inherent in both the eigenvectors and the rotation tangents of [6].

In this paper, we do not consider measures involving $\nabla \widehat{\phi}_{1}$. Unlike the geometry captured by $\nabla \widehat{\phi}_{2}$ and $\nabla \widehat{\phi}_{3}$, tensor rotations around $\boldsymbol{e}_{1}$ do not reflect a change in the fibre tangent direction. Rather, they capture rotations of the diffusion profile around the fibre tangent. Such rotations may be particularly sensitive to noise, and their biological meaning is not immediately clear. We thus leave their study for future work.

\section{$3 \quad$ Experiments and Results}

\subsection{Synthetic Data Validation}

We computed the curving (4) and dispersion (5) indices on the synthetic dataset shown in Fig. 2(a). It is divided in three vertical regions of rotation, one for each eigenvector. Eigenvalue mean and variance are constant throughout the field, and mode [6] increases smoothly from top to bottom. The results are shown in Fig. 2(e,f). As expected, the fanning tensor configurations in the central and 


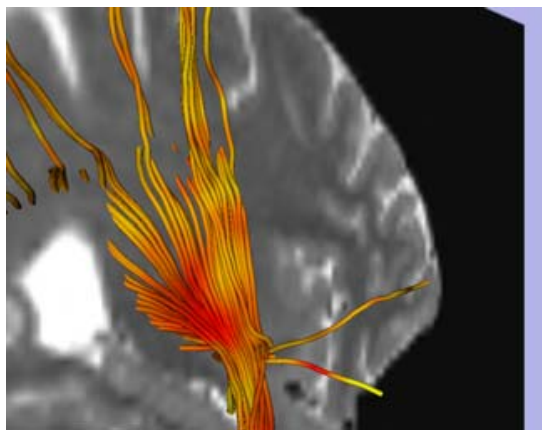

(a)

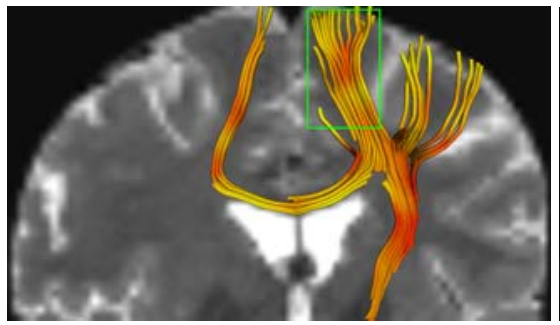

(c)

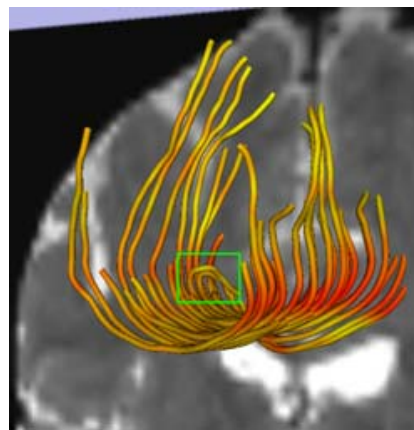

(b)

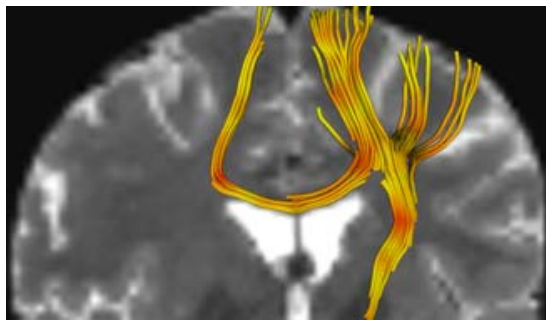

(d)

Fig. 3. Streamline DT tractography colored by dispersion index (a,c) and curving index $(b, d)$. Red indicates high index values. The T2-weighted image is shown in the background for reference. Note the consistent behaviour of the pre-computed dispersion and curving indices with respect to the tractography results. The regions in the green boxes are discussed further in the text. Some of the strong dispersion regions in the internal capsule (c) are due to fibre fanning orthogonal to the image plane.

right-hand side vertical bands have a high dispersion index. The immediately adjacent regions exhibit a high curving index, which is consistent with the underlying geometry. Note that the two measures are not mutually exclusive, and some locations can have both high dispersion and high curving indices.

\subsection{In-vivo Data Validation}

Diffusion-weighted images were acquired on a 3T scanner (General Electric Company, Milwaukee, WI, USA) using an echo planar imaging (EPI) sequence, with a double echo option to reduce eddy-current related distortions. To reduce the impact of EPI spatial distortion, an 8 Channel coil and ASSET with a SENSEfactor of 2 were used. The acquisition consisted in 51 directions with $b=900$, and 8 baseline images with $\mathrm{b}=0$. The scan parameters were: $\mathrm{TR}=17000 \mathrm{~ms}, \mathrm{TE}=78$ $\mathrm{ms}, \mathrm{FOV}=24 \mathrm{~cm}, 144 \times 144$ encoding steps, $1.7 \mathrm{~mm}$ slice thickness. A total of 85 axial slices covering the whole brain were acquired.

The geometric indices were computed only in voxels where the FA value was above 0.2 , in order to avoid tensors with uncertain eigenvector directions, and 


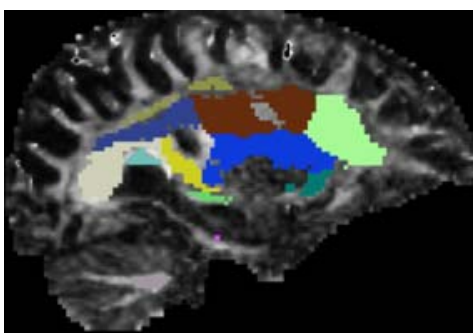

(a)

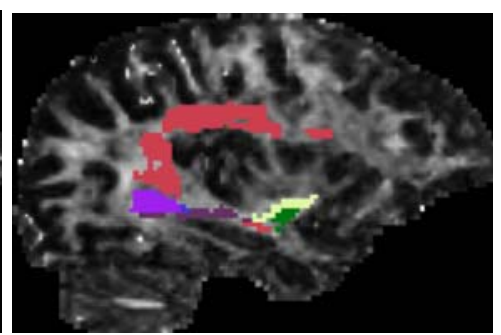

(b)

Fig. 4. Sagittal slices through a patient's right hemisphere, with the ROIs overlaid on an FA map. The results reported in Fig. $5(\mathrm{a}, \mathrm{b})$ pertain to the frontal corona radiata ROI, shown in light green in (a). Those presented in Fig. [5 (c,d) pertain to a segmentation of the uncinate, which combines the light and dark green ROIs in (b). Note the ROIs are $3 \mathrm{D}$ volumes, only a slice through which is visible here.

CSF or grey matter voxels. Prior to computation of the geometrical indices, tensors were normalized by dividing by tensor norm (see e.g. 6] for a definition of tensor norm). After computation of the indices, standard streamline tractography [8] was run to help visualize in three dimensions the behaviour of the indices relative to well-known fibres. The resulting tracts were colored with the pre-computed scalar indices, as shown in Fig. 3. Note how the dispersion index captures the local fanning of fibres passing through the internal capsule in Fig. 3 (a). The fibres run parallel in the bottom part of the green box in Fig. 3(c) and the dispersion index is low. In the upper part of the box, however, the fibres fan, which is characterized by higher index values. While assessing visually these results, it is important to remember that fibre dispersion is a $3 \mathrm{D}$ phenomenon.

Note how the curving index results in Fig. 3(b,d) are consistent with the geometry of the recovered fibre tracts. An exception is seen in the small region enclosed by a green box in (b), where the low index values do not appear to match the geometry of the strongly curved fibre. It appears here that the tractography algorithm wrongly "jumped" onto the cingulum after initially following a callosal fibre. This example shows how our geometrical indices could potentially be used as priors to direct tractography and remove incorrect paths.

\subsection{Group Study on Schizophrenia}

Diffusion MRI data from 20 normal controls (NC) and 23 schizophrenic patients (SZ) was acquired and preprocessed as described in Section 3.2. The deep white matter structures were segmented in regions of interest (ROI) by registering the ICBM-DTI-81 atlas [9] with the diffusion baseline of each subject using nonlinear registration [8].

We computed the dispersion (5) and curving (44) indices in ROIs that segment the frontal part of the corona radiata $(\mathrm{CR})$, as well as the uncinate fasciculus (UN), in both hemispheres. A slice through each ROI in one patient is illustrated 


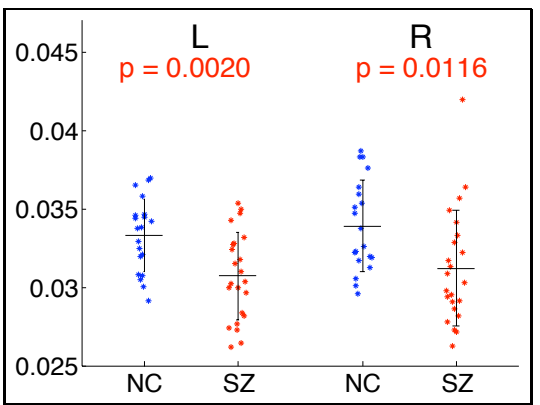

(a) Dispersion, frontal part of CR.

(c) Dispersion, uncinate.

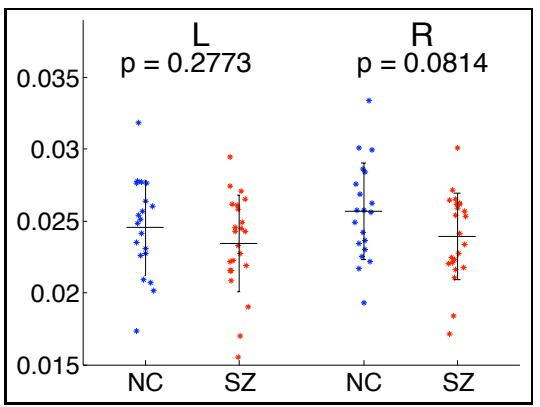

(b) Curving, frontal part of CR.

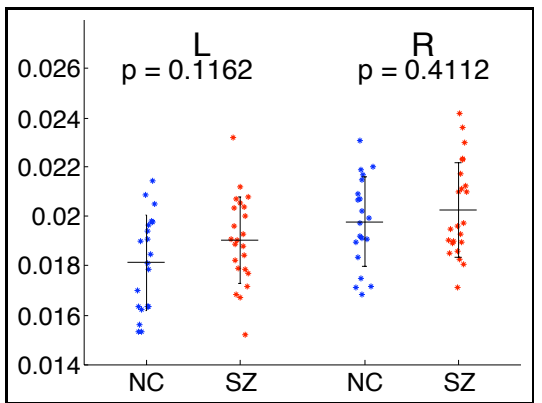

(d) Curving, uncinate.

Fig. 5. Comparison between NC and SZ through scatter plots of mean index value for each individual. Top row: results from the frontal part of the corona radiata. Bottom row: results from the uncinate fasciculus. Left column: dispersion index. Right column: curving index. Blue: NC. Red: SZ. Horizontal black lines: population mean. The error bars indicate \pm 1 standard deviation. L: left hemisphere. R: right hemisphere. The p-values are computed with a paired-value T-test, and those that satisfy a significance threshold of 0.05 are given in red font.

in Fig. 4. The CR is characterized by dispersion, whereas the UN is a curving tract. We thus hypothesized that the dispersion index in the CR case and the curving index in the UN case may reveal certain population differences. The results are presented in Fig. 5. The difference between the NC and SZ populations is significant in the case of the dispersion values in the frontal CR, shown in Fig. 5(a). The curving index comparison in Fig. 5(b,d) shows a trend towards the SZ group having higher index values in the UN, and lower values in the CR. Thus, the population differences are reflected mainly in the dispersion index in the frontal CR. As for the curving index, it reveals an interesting left vs. right asymmetry in the UN.

These results are preliminary, and further study is required before clinical significance claims can be made. For instance, a better ROI definition scheme may be required. Nevertheless, these results serve as a proof of concept and demonstrate that geometrical indices can recover population trends. 


\section{Conclusion}

This paper introduced novel scalar indices of white matter dispersion and curving. Since they are computed directly from the tensor field, without requiring prior tractography, these indices simplify the geometrical analysis of white matter and make it insensitive to possible tractography errors. Traditionally, clinical DTI studies focus on anisotropy, but our results demonstrate that geometrical measures may also be important. Clearly, our region-based comparison results depend on the quality of white matter segmentation. We will address this issue as part of future clinical comparison studies. In work not reported here, we experimented with in-vivo datasets where all tensors with anisotropy higher than a threshold were transformed to a cylindrical shape, by assigning the same eigenvalues to all such tensors, with the idea of removing the influence of tensor shape on the geometrical index values. The results were surprisingly similar to those reported here, and will be discussed in future extensions of this work. Finally, we intend to address applications in fibre tractography and DT image registration, as well as the extension of our indices to a multi-scale framework.

Acknowledgments. Work supported by NIH grants R01MH074794, R01MH50740, K05MH070047, P50MH080272-01, P41RR13218, U54EB005149, U41RR019703, Department of Veteran Affairs Merit Awards, VA Schizophrenia Center.

\section{References}

1. Rovaris, M., Filippi, M.: Diffusion tensor MRI in multiple sclerosis. J. Neuroimaging $17(\mathrm{~s} 1), 27 \mathrm{~s}-30 \mathrm{~s}(2007)$

2. Kubicki, M., McCarley, R., Westin, C.F., Park, H.J., Maier, S., Kikinis, R., Jolesz, F.A., Shenton, M.E.: A review of diffusion tensor imaging studies in schizophrenia. J. Psychiatric Research 41, 15-30 (2007)

3. Pajevic, S., Aldroubi, A., Basser, P.J.: A continuous tensor field approximation of discrete DT-MRI data for extracting microstructural and architectural features of tissue. J. Magn. Resonance 154, 85-100 (2002)

4. Lin, C.P., Tseng, W.Y.I., Cheng, H.C., Chen, J.H.: Validation of diffusion tensor magnetic resonance axonal fiber imaging with registered manganese-enhanced optic tracts. NeuroImage 14, 1035-1047 (2001)

5. Batchelor, P.G., Calamante, F., Tournier, J.D., Atkinson, D., Hill, D.L.G., Connelly, A.: Quantification of the shape of fiber tracts. Magn. Res. in Medicine 55, 894-903 (2006)

6. Kindlmann, G., Ennis, D.B., Whitaker, R.T., Westin, C.F.: Diffusion tensor analysis with invariant gradients and rotation tangents. IEEE Trans. Medical Imaging 26(11), 1483-1499 (2007)

7. Savadjiev, P., Zucker, S.W., Siddiqi, K.: On the differential geometry of 3D flow patterns: Generalized helicoids and diffusion MRI analysis. In: Proc. IEEE Intl. Conf. on Computer Vision, ICCV 2007 (2007)

8. http://www.slicer.org

9. http://loni.ucla.edu/Atlases/ 